IMPLICACIONES SOCIOEDUCATIVAS DEL CONFINAMIENTO SOCIAL POR LA PANDEMIA COVID-19. ESTUDIO DE OPINIÓN DE ESTUDIANTES DE LA UNIVERSIDAD INTERCULTURAL DE TABASCO.

\title{
IMPLICACIONES SOCIOEDUCATIVAS DEL CONFINAMIENTO SOCIAL POR LA PANDEMIA COVID-19. ESTUDIO DE OPINIÓN DE ESTUDIANTES DE LA UNIVERSIDAD INTERCULTURAL DE TABASCO
}

\section{SOCIOEDUCATIONAL IMPLICATIONS FROM SOCIAL DISTANCING DUE TO THE COVID- 19 PANDEMIC. STUDENTS' OPINION STUDY FROM UNIVERSIDAD INTERCULTURAL DE TABASCO}

\begin{abstract}
Jesús García Guadalupe*, José Ramón Contreras De la Cruz**,

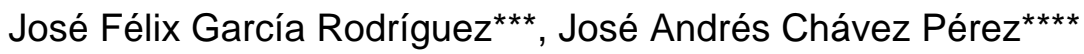

\footnotetext{
* Licenciado en Lingüística y Literatura Hispanoamericana por la Benemérita Universidad Autónoma de Puebla. Profesor de Tiempo Completo de la Universidad Intercultural del Estado de Tabasco. Email: jesus.garcia@uiet.edu.mx, ORCID: http://orcid.org/0000-0003-2603-6311.

** Maestro en Administración y Políticas Públicas por el Instituto de Administración Pública de Tabasco A.C. Profesor de Tiempo Completo de la Universidad Intercultural del Estado de Tabasco. Email: jose.ramon@uiet.edu.mx, ORCID: http://orcid.org/0000-0002-6502-7883.

*** Doctor en Finanzas Públicas por la Facultad de Economía de la Universidad Veracruzana; ProfesorInvestigador de la Universidad Juárez Autónoma de Tabasco. ORCID: http://orcid.org/0000-0002-73191472.

**** Doctor en Administración por la Universidad del Sur, Tuxtla Gutiérrez Chiapas. Profesor de Tiempo Completo de la Universidad Intercultural del Estado de Tabasco. Email: andres.chavez@uiet.edu.mx, ORCID: http://orcid.org/0000-0002-8307-4691.
}

Dirección para correspondencia: jfgr55@hotmail.com 
IMPLICACIONES SOCIOEDUCATIVAS DEL CONFINAMIENTO SOCIAL POR LA PANDEMIA COVID-19. ESTUDIO DE OPINIÓN DE ESTUDIANTES DE LA UNIVERSIDAD INTERCULTURAL DE TABASCO.

\section{RESUMEN}

OBJETIVO: Conocer la opinión de los estudiantes de la Universidad Intercultural de Tabasco (UIET), acerca del impacto de la pandemia en el proceso educativo en su modalidad a distancia durante el semestre febrero-junio 2020, adoptada como estrategia educativa emergente a partir de las medidas de sana distancia y confinamiento.

MATERIAL Y MÉTODO: El enfoque de la investigación es cualitativo y de naturaleza hermenéutica, sustentado en la aplicación de entrevistas semiestructuradas a 70 estudiantes de las siete licenciaturas que ofrece la institución.

RESULTADOS: Esta experiencia permitió interpretar como asumen los estudiantes la crisis sanitaria, y la manera en que ha afectado su vida académica y familiar, así como generar recomendaciones para la mejora de la práctica educativa en eventuales contextos emergentes.

CONCLUSIONES: La presencia del COVID-19 y el confinamiento prolongado han tenido efectos significativos en el proceso de enseñanza - aprendizaje de los jóvenes universitarios, en especial de zonas rurales indígenas, que han afectado negativamente su rendimiento escolar y en algunos casos, sus posibilidades de continuidad y conclusión de sus estudios.

PALABRAS CLAVE: Educación intercultural. Educación a distancia. Pandemia. Covid-19. Distanciamiento social.

\section{ABSTRACT}

OBJECTIVE: To know the students' opinion from Universidad Intercultural de Tabasco (UIET), about the impact of the pandemic in the educative process in the Distance Mode during the school term: February-June 2020, adopted as an emerging educative strategy as the healthy distance and seclusion measure.

MATERIAL AND METHOD: The approach of the study is qualitative and hermeneutic. Based on semi-structured interviews to 70 students from the seven careers offered in the institution. 
IMPLICACIONES SOCIOEDUCATIVAS DEL CONFINAMIENTO SOCIAL POR LA PANDEMIA COVID-19. ESTUDIO DE OPINIÓN DE ESTUDIANTES DE LA UNIVERSIDAD INTERCULTURAL DE TABASCO.

RESULTS: This experience allowed the interpretation of the way students assume this health crisis and the way it has affected their academic and family life; and thus generate suggestions in order to improve the educative practice in emerging scenarios.

CONCLUSIONS: The presence of COVID-19 and the prolongued seclusion have had significant effects in the teaching learning process in college students, specially in indigenous areas, which have negatively affected their academic performance and in some case, their continuity and their career completion.

KEY WORDS: Intercultural Education. Distance Mode Program. Pandemic. Covid-19. Social Distancing.

\section{INTRODUCCIÓN}

En marzo de 2020, las autoridades sanitarias y educativas de México, así como de otros países del mundo decretaron el cierre de las escuelas y los centros de trabajo, para evitar a toda costa el contagio masivo por la nueva enfermedad COVID-19 ocasionada por el coronavirus SARSCoV2, cuya alta diseminación amenazaba con colapsar los servicios hospitalarios. Solo siguieron funcionando los servicios esenciales como los de salud, alimentos, transporte, agua, electricidad, construcción, saneamiento, limpia, registro civil, entre otros. La UNESCO reportó que, al mes de abril de este año, 191 países habían cerrado sus escuelas y el 93\% de su matrícula ya no estaba asistiendo a sus centros escolares (Castillo, 2020). Este fenómeno es único como nunca en la historia reciente, en que se han detenido las actividades en todo el mundo y se pronostica que esta crisis de salud, tenga secuelas en la economía, la producción de alimentos y en la seguridad en un periodo entre dos a cinco años (CEPAL, 2020).

La Asociación Nacional de Universidades e Instituciones de Educación Superior (ANUIES), que agrupa a 196 universidades públicas y privadas de México, desde el inicio de la contingencia tomó algunas medidas; por ejemplo, emitió recomendaciones para enfrentar la pandemia y para el retorno a la nueva normalidad a través del documento "Hacia la construcción colectiva de la nueva normalidad en la educación superior" (ANUIES, 2020 a) y proveyó de herramientas útiles 
IMPLICACIONES SOCIOEDUCATIVAS DEL CONFINAMIENTO SOCIAL POR LA PANDEMIA COVID-19. ESTUDIO DE OPINIÓN DE ESTUDIANTES DE LA UNIVERSIDAD INTERCULTURAL DE TABASCO.

para el trabajo académico en su página institucional (ANUIES, 2020b). En cuanto a la Universidad Intercultural del Estado de Tabasco (UIET), desde el año 2005 que se fundó, no se había presentado un cambio tan drástico en el proceso formativo de los estudiantes universitarios como el que se vive con el COVID-19.

Desde marzo y hasta el cierre del ciclo escolar a finales de junio del mismo año, las actividades escolares se realizaron a distancia; este cambio sorpresivo, tendrá efectos inmediatos y de mediano plazo en la vida académica de los estudiantes universitarios mexicanos, en especial los provenientes de familias con bajos niveles socioeconómicos, y más todavía en estudiantes provenientes de entornos multiculturales. Este estudio se realizó al cierre del semestre febrerojunio de 2020, con el propósito de conocer algunos efectos del confinamiento por COVID-19 en la vida académica de los estudiantes de la UIET, así como conocer su percepción al respecto, con el fin de mejorar la práctica educativa. Para efecto del análisis, nos centraremos en tres aspectos: La inequidad y la educación superior; la resiliencia como respuesta a la crisis, y los cambios en el proceso educativo universitario.

\section{Inequidad y educación superior}

La pandemia por COVID-19 hizo visible la desigualdad en nuestras sociedades, en especial en los países Latinoamericanos, tal como lo reportan diversos autores; como Vijil (2020) en Nicaragua, Vivanco (2020) y Vallejo (2020) en Ecuador, Failache (2020) en Uruguay, Tzoc (2020) en Guatemala, y Dietz, (2020), Mérida y Acuña (2020) Buendía (2020), Acosta (2020) y CONEVAL (2020) en México. Esta desigualdad se materializó en la ausencia de los medios para continuar la formación en casi todos los niveles educativos; la UNESCO, UNICEF, FAO, OIT y otros organismos internacionales coinciden que en los próximos meses y posiblemente años, la crisis de salud, económica y social se verá exacerbada y la brecha de desigualdad se incrementará (Renna, 2020; Acosta, 2020 y OIT, 2020).

EI CONEVAL (2020), menciona que esta crisis sanitaria tendrá impactos inevitables en el corto y mediano plazo en México, en aspectos como la economía, el comercio, el empleo y en el bienestar de la población. La desigualdad en México se refleja en el 49\% de su población en pobreza y con una población con presencia crónica de enfermedades como diabetes, desnutrición, obesidad, hipertensión, así como la dependencia de ingresos de más de la mitad de las familias mexicanas del empleo informal; 24.7 millones de personas (19.8\%) con falta de 
IMPLICACIONES SOCIOEDUCATIVAS DEL CONFINAMIENTO SOCIAL POR LA PANDEMIA COVID-19. ESTUDIO DE OPINIÓN DE ESTUDIANTES DE LA UNIVERSIDAD INTERCULTURAL DE TABASCO.

acceso al agua, a servicios básicos y con hacinamiento en sus viviendas (CONEVAL, 2018); estos últimos factores complican la puesta en marcha de las medidas para contener la epidemia y vulneran el derecho humano al agua y a la salud, al no poder cumplir con las medidas de higiene básica de lavarse las manos con agua y jabón y al aislamiento domiciliario de los positivos y sospechosos (Mérida y Acuña, 2020); también se observa esta desigualdad en el acceso a la educación, en especial a la educación superior; Acosta (2020) reporta que este país es de los peores calificados en rezago educativo acumulado de la OCDE, por ejemplo en educación básica, el 30\% de los niños que ingresan a la primaria no concluyen su secundaria; y es el último lugar de 35 países de la ODCE en cobertura en educación superior, por el orden del $39 \%$, la principal causa en ambos casos es el bajo nivel socioeconómico de las familias de origen de los estudiantes.

Por su parte, Mérida y Acuña (2020) alertan sobre el riesgo de inequidad en el acceso a los programas educativos del nivel básico en algunas entidades federativas con altos niveles de marginalidad, por ejemplo el programa "Aprende en casa" federal y sus contrapartes de los gobiernos estatales, al no considerar la difícil y nula conectividad a televisión, internet o radio de algunas regiones del país, lo que puede acarrear bajos niveles de aprendizajes, incrementar la brecha educativa, la desigualdad y la injusticia social. De igual manera, Dietz (2020), advierte que la desigualdad se verá incrementada por la pandemia, pero más aún en el acceso a la educación en contextos de diversidad cultural; por ello, las escuelas y universidades interculturales que están enclavadas en estas regiones multiculturales pueden jugar un papel importante para contribuir a revertir estas desigualdades. Algunos expertos señalan, que la educación superior sufrirá cambios drásticos al pasar de la formación presencial a la formación en línea, igual que algunas áreas del desarrollo económico, como el trabajo en línea, la industria y el turismo de negocio y placer; (Vijil, 2020), así como la reducción de la internacionalización y movilidad estudiantil (Mérida y Acuña, 2020).

\section{La resiliencia como respuesta a la crisis}

Los pueblos originarios de América, en especial de los países latinoamericanos están siendo impactados por esta crisis de salud en diferentes escalas, Hooker (2020), refiere que “... han visto trastocados diferentes esferas de su vida interna al verse obligados a hacer cambios rápidos y drásticos en la organización de sus formas de vida que han afectado su espiritualidad, el respeto, las formas de enfrentar la enfermedad, la muerte y el luto y en general el equilibrio y 
IMPLICACIONES SOCIOEDUCATIVAS DEL CONFINAMIENTO SOCIAL POR LA PANDEMIA COVID-19. ESTUDIO DE OPINIÓN DE ESTUDIANTES DE LA UNIVERSIDAD INTERCULTURAL DE TABASCO.

armonía de su entorno" (p. 2). Sin embargo, se reconoce que, en estas sociedades, están emergiendo nuevas capacidades relacionadas con la solidaridad, la resiliencia y la inteligencia emocional para aprender de los eventos y sucesos por muy dolorosos que sean y siempre retornar a la tierra para cuidarla y convivir con ella. Siguiendo a esta misma autora, se señala que las universidades interculturales en Latinoamérica, están experimentando junto con las comunidades locales nuevas formas de enfrentar la crisis, de por sí con entornos complejos y con las dificultades que ya se tenían antes de la pandemia, que incluía disminución de financiamiento, crecimiento sin calidad, inequidad en el acceso a la infraestructura y servicios en relación a las otras instituciones del sistema educativo superior.

Dietz (2020), menciona la estrategia de comunidades indígenas de Veracruz para responder de manera diferente al riesgo de la pandemia, en lugar del "Quédate en casa" implementado de manera obligatoria por gobierno federal, ellos aseguraron los accesos a su comunidad para evitar ingresos foráneos de personas, aun de sus familiares migrantes, en un claro mensaje de colectividad "quédate en comunidad", que -según menciona-, bien puede también significar en el aspecto educativo, aprender en comunidad. El mismo autor, nos hace recordar que los pueblos originarios americanos han demostrado por siglos que son expertos en resiliencia, al enfrentar todo tipo de catástrofes y pandemias con la unidad comunitaria, el consejo de sus sabios y ancianos y su memoria biocultural, no es dudar que saquen lo mejor para enfrentar esta nueva pandemia.

Por otra parte, Tzoc (2020), menciona que los pueblos indígenas mesoamericanos tienen un punto de vista multidimensional de la enfermedad y de la muerte y que enfrentan de manera diferente la crisis. De entrada, se relacionan con respeto con todos los seres vivos, aun los que causan sufrimiento y enfermedad; tienen la noción que todo se mueve dentro de periodos de orden y caos y que es el deber del ser humano buscar restablecer este orden y armonía con la naturaleza; que esta pandemia es una oportunidad de valorar la vida en comunidad y en familia y buscar respuestas locales, aprender, reconciliarse con la tierra y la naturaleza y darle sentido a la vida. En el mismo sentido, Vallejo (2020) ha estado dando seguimiento al desarrollo de la pandemia entre pueblos amazónicos del Ecuador y ha encontrado que tienen formas locales de fortalecer su sistema respiratorio y sus defensas con plantas de la selva; sin embargo, el riesgo de contagio por el virus SARS-CoV2 es alto, ya que por provenir de una especie silvestre el ser humano no tiene defensas para ello. Los casos se incrementan y ante la ausencia de los servicios de salud del estado, la migración juvenil entre la ciudad y el campo y la presencia de GARCÍA-GUADALUPE J., CONTRERAS-DE LA CRUZ J. R., GARCÍA-RODRÍGUEZ J. F., CHÁVEZ-PÉREZ J. A. 
IMPLICACIONES SOCIOEDUCATIVAS DEL CONFINAMIENTO SOCIAL POR LA PANDEMIA COVID-19. ESTUDIO DE OPINIÓN DE ESTUDIANTES DE LA UNIVERSIDAD INTERCULTURAL DE TABASCO.

terceros por actividades extractivas, mineras, de petróleo y explotación forestal, temen por la pérdida de los ancianos con sus saberes y memoria histórica.

La OIT en su encuesta mundial a jóvenes 2020 (OIT, 2020), ha encontrado que la afectación por el COVID-19 y el confinamiento ha sido sistemática, profunda y desproporcionada para las y los jóvenes entre 18 y 29 años, y que tendrá secuelas en el tiempo. Documenta los efectos de la pandemia en cuatro ámbitos: empleo e ingresos, educación y formación, bienestar mental y disminución de sus derechos, estos últimos como la libertad de culto, el acceso a la información, la participación ciudadana, y el acceso a una vivienda digna. En la UIET, esta pandemia alteró los programas de estudio y la planeación de las clases, al suspenderse las sesiones presenciales se presentaron situaciones imprevistas a las que no se estaba acostumbrado. El docente y los estudiantes se vieron coartados por el tiempo y el espacio. Este confinamiento empezó con el encierro y el distanciamiento social y provocó una ruptura en la dinámica escolar y en la cultura de la convivencia escolar. La tarea educativa se volvió más compleja, al atender a los estudiantes universitarios y las actividades escolares propias de los hijos, tanto para ellos como para los docentes universitarios. Pese a las dificultades, complejidades y carencias, los estudiantes han enfrentado estos escenarios con el acompañamiento de sus familias y sus profesores. La formación desarrollada en su estancia en la universidad y su propia historia de vida de proveniencia de familias acostumbradas a la lucha diaria por la sobrevivencia, les ha permitido enfrentar con relativo éxito estas dificultades.

\section{Los cambios en el proceso educativo universitario}

Lo que al principio se suponía, sería una interrupción temporal de las actividades escolares y productivas, se fue ampliando hasta un confinamiento prolongado. La continuación de las actividades educativas a distancia, pensadas como una medida temporal hasta que se restableciera la presencialidad, con el paso de las semanas se fue tornando en definitiva y puso en aprietos a la mayor parte de los sistemas educativos, tanto del sistema básico para niños y adolescentes como para los jóvenes del nivel universitario y obligó a plantearse transformaciones en el paradigma presencial-virtual de cara a los nuevos tiempos. Acosta (2020) señala que la inactividad académica de los estudiantes que no tienen los medios, puede llevar a disminuir en un 10\% los aprendizajes y afectar el paso a los grados sucesivos, el egreso y la empleabilidad, esta es una de las principales preocupaciones de las instituciones de 
IMPLICACIONES SOCIOEDUCATIVAS DEL CONFINAMIENTO SOCIAL POR LA PANDEMIA COVID-19. ESTUDIO DE OPINIÓN DE ESTUDIANTES DE LA UNIVERSIDAD INTERCULTURAL DE TABASCO.

educación superior que atienden jóvenes provenientes de familias de bajos niveles socioeconómicos, entre ellas las universidades interculturales.

Cotino (2020) lo menciona desde un enfoque del derecho a la educación, al sugerir convertir la educación virtual, de un salvavidas en la crisis sanitaria a una oportunidad de cambios que aproveche mejor los recursos digitales; apunta que, aunque la educación presencial es necesaria y en muchos aspectos superior a la educación en línea, por ahora ésta última es la que va a permitir la continuidad de los aprendizajes de los estudiantes en el mundo. Este mismo autor, recomienda considerar el decálogo de edula@ab de la Universidad Oberta de Cataluña (EDUL@AB, 2020) para un mejor aprovechamiento de las herramientas digitales en la educación. Por su parte, Acosta (2020) destaca que en el caso del nivel superior, cada universidad de acuerdo a sus medios disponibles ha respondido de manera diferente ante la pandemia; por ejemplo, la Universidad Nacional Autónoma de México (UNAM) y la Universidad Autónoma Metropolitana (UAM), han implementado programas emergentes, que pretenden mitigar el impacto negativo de la pandemia; en el caso de la UAM, la institución ha otorgado a sus estudiantes de bajo nivel socioeconómico, tabletas y tarjetas para su uso en el trimestre y ha implementado el Proyecto Emergente de Enseñanza Remota (UAM, 2020).

Por otro lado, Gazzo (2020), recomienda dejar de mirar el ciberespacio de la educación virtual como una caja negra o dispositivo para subir archivos de información que el estudiante descargará y aprovechará, propone en cambio, desarrollar contenidos concretos pero profundos, espacios colaborativos y mantener la interacción docente-estudiante como un hilo conductor durante todo el proceso. La misma autora, hace referencia a la reivindicación del papel de la generación de millennials que hasta hace unas semanas eran reprimidos, castigados y señalados por depender de los dispositivos electrónicos, justo en este viraje social repentino empujado desde la biología por un organismo diminuto, es que sus habilidades serán de gran utilidad.

La desescolarización forzada y la migración a un sistema de educación virtual a todas luces monocultural parece ser un retroceso a los avances logrados en las últimas dos décadas de la interculturalidad educativa, según lo expresa Dietz (2020); sin embargo, bien puede ser la oportunidad de promover cambios profundos en ese sentido en el sistema educativo. Por ejemplo, siguiendo al mismo autor, algo positivo en esta pandemia es el papel importante que ha asumido la familia en la educación: abuelos, padres, tíos y tías, hermanos y hermanas, 
IMPLICACIONES SOCIOEDUCATIVAS DEL CONFINAMIENTO SOCIAL POR LA PANDEMIA COVID-19. ESTUDIO DE OPINIÓN DE ESTUDIANTES DE LA UNIVERSIDAD INTERCULTURAL DE TABASCO.

contribuyen al logro educativo de niñas, niños, adolescentes y jóvenes, y no sólo en casa sino también en las labores productivas y en la comunidad; no obstante, debe reconocerse que también ha propiciado un aumento en los niveles de violencia intrafamiliar. En este sentido, Porlan (2020) va al extremo en su análisis crítico de los sistemas educativos actuales a la luz de la actual crisis de salud; menciona que la oportunidad está en dar un viraje a la educación con o sin pandemia, con la corrección de múltiples carencias detectadas; considera anacrónica a la educación actual en la mayoría de los países, con una urgencia de cambios sustanciales; no puede seguir siendo una simple transmisión de información del docente al estudiante, sugiere la necesidad de ejercitar la actividad mental del sujeto, y que los contenidos sean relevantes, con significado, sistémicos y con conexión entre las diferentes disciplinas.

Lo cierto es, que esta crisis sanitaria puede ser una gran oportunidad para impulsar propuestas educativas que parecían olvidadas, de difícil aceptación y hasta utópicas, que propicien la formación integral de seres humanos más sensibles hacia la naturaleza, a la urgencia de cambios para mitigar el impacto de las crisis provocadas por nuestra especie y que no se habían podido visibilizar por el avasallamiento de los modelos de desarrollo de corte economicista que privilegian el crecimiento y la acumulación del capital. La educación y en especial la educación superior, debe contribuir a plantar cara a las crisis que ya teníamos aun sin pandemia: cambio climático, agotamiento de recursos naturales, hambre e ingobernabilidad en algunas regiones; ante la incertidumbre de la crisis sanitaria, se perfilan por lo menos tres escenarios: que se puedan exacerbar las crisis y por ende la inequidad e ingobernabilidad, que se aproveche esta coyuntura y se generen cambios sustanciales de los cuales todos salgamos beneficiados; o un desafortunado regreso a la "normalidad" como si nada hubiese pasado. Como lo han documentado Tzoc en Guatemala, Vallejo en Ecuador, Dietz en México y Hooker y Castillo en Nicaragua, los conocimientos de los pueblos indígenas, la sabiduría ancestral heredada por generaciones, el respeto a la naturaleza incluida las epidemias como una respuesta de la madre tierra a un desequilibrio y la resiliencia para enfrentar desafíos extremos a la que han estado expuestos, todo ello nos ofrecen alternativas que no hay que demeritar, que habrá que escuchar a través de nuestros estudiantes que provienen de estos pueblos originarios. 
IMPLICACIONES SOCIOEDUCATIVAS DEL CONFINAMIENTO SOCIAL POR LA PANDEMIA COVID-19. ESTUDIO DE OPINIÓN DE ESTUDIANTES DE LA UNIVERSIDAD INTERCULTURAL DE TABASCO.

\section{MATERIAL Y MÉTODO}

Estudio de enfoque cualitativo, sustentado en la metodología de investigación narrativa y biografía-narrativa, donde se busca generar en el sujeto estados de reflexión y conciencia de las experiencias vividas, así como aprovechar las subjetividades para identificar y explicar los procesos educativos (Landín y Sánchez, 2019). Los relatos y narraciones dan sentido a la vida de las personas, y la experiencia de quien interviene estos procesos cobra sentido en la medida que hay una voz que es escuchada (Sparkes y Devís, 2007). La sistematización de las experiencias puede generar nuevos conocimientos y apoyar la toma de decisiones apropiadas en lo individual y colectivo y así transformar la práctica y el contexto. En los estudios sobre juventud y conflicto de Pinilla y Lugo (2011), los jóvenes expresan sus experiencias, comparten su mundo desde sus construcciones sociales y culturales, desde la complejidad de sus contextos. Justo en éstos, cuando están sometidos a presión externa debida a conflictos, la crisis puede servir de motor para generar iniciativas, soluciones a problemas y crear redes de apoyo de manera creativa. Este es el caso de los jóvenes universitarios de la UIET.

Esta investigación revela sus ideas, sus vivencias y preocupaciones sobre el entorno donde viven, y revelan cómo enfrentan la crisis de salud, el riesgo, el aislamiento forzado y, en algunos casos, la pena, la muerte y la enfermedad de un familiar debido a la pandemia. Para el levantamiento de la información se aplicó un instrumento de investigación de forma aleatoria a 70 estudiantes de las siete licenciaturas que ofrece la UIET; se analizaron 10 ítems, buscando conocer la percepción sobre los cambios en el proceso de su formación profesional durante este periodo de trabajo a distancia derivado por el confinamiento de la pandemia COVID-19.

\section{RESULTADOS Y DISCUSIÓN}

Sistematizar esta experiencia significa poner en el centro a los actores principales del proceso educativo. La intención es escuchar la voz de lo que ellos sintieron, de cómo vivieron este episodio abrupto que movió los esquemas de su vida universitaria. En sus narraciones, los estudiantes expresan sus experiencias en el proceso académico, aislados de sus compañeros, de sus maestros y de su aula de trabajo. En una de las preguntas, indicaron sus principales aprendizajes de este periodo vivido: "Aprendí como sobrellevar las problemáticas; trabajar en línea ha sido muy bueno ya que mejora más la calidad de comunicación, hubo problemas, pero pude sobrellevarlos" (Estudiante 1, 2020). 
IMPLICACIONES SOCIOEDUCATIVAS DEL CONFINAMIENTO SOCIAL POR LA PANDEMIA COVID-19. ESTUDIO DE OPINIÓN DE ESTUDIANTES DE LA UNIVERSIDAD INTERCULTURAL DE TABASCO.

Como se aprecia, un aspecto positivo es que esta pandemia los llevó a enfrentar el problema, fue un reto inesperado. Este comentario, indica que tuvo un agrado por este tipo de ejercicio de trabajar en línea. Incluso, este discurso para el docente representa un gran aliento, o como el siguiente relato que vislumbra cierta autonomía en el estudio. "El no esperar a que el maestro solucionara o enviara la información necesaria para realizar algunas de las actividades. El organizar de manera provechosa el tiempo de modo que diera tiempo de cumplir con las diversas actividades". (Estudiante 2, 2020).

Esto lleva a pensar que tuvo los recursos para poder establecer la comunicación vía internet o telefónica, pues lo ve como algo positivo a pesar de la situación adversa; no se detiene ante el problema, al contrario, lo ve como un área de oportunidad.

Por otro lado, hubo quienes manifestaron lo contrario, la pesadez para llevar a cabo estas actividades escolares a lo que o estaban acostumbrados, a verse, a dialogar frente a frente. "Un poco complicado ya que la comunicación entre los maestros fue muy regular ya que hubo ocasiones que en la cual no fue muy entendible, pero a pesar de la pandemia y la educación a distancia se pudo lograr terminar nuestro semestre." (Estudiante 3, 2020).

Esta nota evidencia que por una parte el estudiante pone sus esfuerzos, pero la otra parte le corresponde al tutor, que tiene que buscar la mejor herramienta educativa para poder generar el interés del estudiante y que por supuesto, es una atención individual que, si bien es desgastante, también permite conocerlo de una manera más propia, pues tiene que trabajar sólo y puede ver aún mejor sus limitantes, tecnologías educativas y personales.

Otro modo de vivir este trance académico fue justamente lo que ocurre con aquellos estudiantes que no tienen acceso al recurso tecnológico, ni mucho menos experiencia en el manejo del recurso virtual, aunque lo mismo ocurre con algunos de los docentes; la diferencia es que docentes si tienen más posibilidades para invertir en un aparato tecnológico.

"La verdad, para mí fue algo complicado y estresante, ya que casi no sabía utilizar algunos programas e igual no contaba con una computadora propia, además en la comunidad en la que vivo es muy lejana de la villa y no cuenta con mucha señal y menos con un cíber" (Estudiante 4, 2020). 
IMPLICACIONES SOCIOEDUCATIVAS DEL CONFINAMIENTO SOCIAL POR LA PANDEMIA COVID-19. ESTUDIO DE OPINIÓN DE ESTUDIANTES DE LA UNIVERSIDAD INTERCULTURAL DE TABASCO.

No sólo es el problema de que tenga o no una computadora, sino más bien es el acceso al servicio de internet. Las características geográficas evidencian las limitantes de factores externos que impiden que los estudiantes puedan responder a las exigencias académicas virtuales.

"Hubo algunas complicaciones en el caso de abarcar algunos de los temas a tratar ya que en algunos casos el docente solo nos mandaba lo que debíamos investigar y no daba algún tipo de explicación antes o después. Aparte de eso, se extrañaba la presencia de algunos compañeros de clase, y el poder interactuar entre nosotros en ocasiones se tornaba complicado por dificultades de conexión" (Estudiante 5, 2020).

Se refleja aquí la diversidad de las apreciaciones, este juicio pone en el centro el papel del docente. Alude al tacto del docente para pensar en el proceso metodológico de cómo guía al estudiante en su aprendizaje. Si fuera presencial, da oportunidad para replantear una duda, sugerencia u opinión, pero en este caso, ¿cómo hacerle? además, el estudiante apela a la interacción, un elemento fundamental en los procesos educativos que permiten un crecimiento colectivo y que así, de pronto, se les despojo de esta forma de trabajo escolar.

Otra de las razones que se encontró en este ejercicio fue la carga personal, adicional a las académicas: "La verdad, fue muy difícil para mí, ya que yo me mantengo sola y ni me daba tiempo hacer mis tareas. Algunas tareas no las entregue por eso creo que me afecto, solo hice lo que pude" (Estudiante 6, 2020).

Las actividades que tiene que ver con otras responsabilidades que implicaron el confinamiento es justamente con el proveer los alimentos, atender la cotidianidad del hogar, situaciones que no se pusieron como elementos determinantes y quizá evaluables de este proceso de aprendizaje.

A pesar de todo, la responsabilidad de los estudiantes también se matizó en sus comentarios, pues la meta es sin duda, obtener una nota positiva para continuar en la formación académica.

"En general, en este semestre se me complico un poco por falta de internet, y así mismo porque también vivo en una comunidad que cuenta con poca señal telefónica y tenía que gastar dinero para hacer recargas y estar al tanto con mis tareas, pero aun 
IMPLICACIONES SOCIOEDUCATIVAS DEL CONFINAMIENTO SOCIAL POR LA PANDEMIA COVID-19. ESTUDIO DE OPINIÓN DE ESTUDIANTES DE LA UNIVERSIDAD INTERCULTURAL DE TABASCO.

así hice todo lo posible por enviar en tiempo y forma mis trabajos para salir bien en el semestre" (Estudiante 7, 2020).

Se nota aquí la voz de la sensibilidad económica al invertir un recurso para cumplir con las actividades escolares. En este relato expresa la manera de cómo vive este episodio:

"En lo personal, fue una labor complicada dado los diferentes papeles que desempeño hasta la actualidad como integrante de una familia numerosa, donde nos vimos afectados por el virus, y lógicamente esta pandemia arrastro la economía del hogar de forma horrorosa, dejando a expensas de muchas cosas, posteriormente la presión de algunas actividades en horarios estrictos fue un factor para un resultado de calificaciones bajas, cabe mencionar que hubo momentos en que no disponía de internet y no se tenía acceso a un cíber abierto" (Estudiante 8, 2020).

Estos datos corroboran las condiciones en que se enfrentaron estas clases virtuales; quienes tuvieron los recursos necesarios para atender las actividades asignadas de los docentes son los que tuvieron éxito, pasaron la prueba, quienes no contaron con los medios necesarios sufrieron el estrés por no responder en tiempo y forma como lo exigía la adecuación del sistema virtual para concluir el semestre.

"En lo personal, tuve muchas dificultades sobre la forma en la que tenía que elaborar mis trabajos [...] estar saliendo de casa pues no ayuda de nada. Pero busque la manera de realizar mis trabajos y sobre todo salvar mis materias, pero en realidad lo más conveniente pues es estar en un aula donde te puedan explicar bien detalladamente" (Estudiante 9, 2020).

Por un lado, se pone en evidencia todo el mecanismo que mueve al estudiante para enfrentar la modalidad virtual en esta pandemia. Desde el que puede hacerlo porque tiene el soporte económico, material, familiar, hasta el que carece de todos los medios para poder salir avante en estas tareas. ¿De qué sirve un confinamiento si tiene que salir de casa a un cíber y permanecer el tiempo necesario para subir sus tareas, o realizar sus investigaciones? Eso el docente no lo visualiza, le interesa que el estudiante responda, entregue sus avances. Por otro lado, esta voz añora la forma presencial para estudiar, refleja la necesidad de un docente para que pueda apoyar su aprendizaje. 
IMPLICACIONES SOCIOEDUCATIVAS DEL CONFINAMIENTO SOCIAL POR LA PANDEMIA COVID-19. ESTUDIO DE OPINIÓN DE ESTUDIANTES DE LA UNIVERSIDAD INTERCULTURAL DE TABASCO.

"[...] este semestre que ya se concluyo fue [...] muy estresante porque es una manera, que no convives con otras personas para compartir ideas y obtener experiencia con ellos como participaciones y diálogos, fue un poco complicado porque faltaba el equipo de internet, aunque fue muy forzoso que haga la contratación, pero de todos modos lo contrate para que yo realice mis actividades para que yo no reprobara el semestre [...]." (Estudiante 10, 2020).

Asimismo, evidenció el sentir de la responsabilidad de la carga académica, convertido en estrés, además, realizó un gasto que no estaba dentro del plan, contratar internet. Todo ello, resulta un factor minimizado, en tanto que algunos estudiantes aparecieron con calificaciones no aprobatorias. Como pudo verse, varios de estos factores no fueron considerados como elementos determinantes en el cumplimiento de las tareas y en la evaluación del desempeño académico de los estudiantes en la UIET.

Los anteriores hallazgos narrativos, aunado a los datos cuantitativos, muestran una parte de la profundidad y complejidad del problema. Esta pandemia sacó a relucir las carencias, las vidas esforzadas y de alta vulnerabilidad de los estudiantes de regiones rurales e indígenas para poder concluir sus estudios superiores.

Asimismo, también muestra la gran responsabilidad que la educación superior tiene para la transformación de las sociedades latinoamericanas, en especial la responsabilidad que tienen las universidades interculturales en las regiones con presencia de diversidad cultural para construir espacios de convivencia en la diversidad, con respeto a la identidad, a los derechos humanos y a los saberes locales.

\section{CONCLUSIONES}

En base a los resultados obtenidos se puede afirmar que la presencia del COVID-19 y el confinamiento prolongado han tenido efectos significativos en el proceso de enseñanza aprendizaje de las y los jóvenes universitarios, en especial de zonas rurales, que han afectado negativamente su rendimiento escolar y en algunos casos, sus posibilidades de continuidad y conclusión de sus estudios.

Es importante considerar la necesidad de modificar las estrategias para el aprendizaje, el desarrollo de contenidos e indicadores de evaluación, que incluya, no solo los conocimientos, 
IMPLICACIONES SOCIOEDUCATIVAS DEL CONFINAMIENTO SOCIAL POR LA PANDEMIA COVID-19. ESTUDIO DE OPINIÓN DE ESTUDIANTES DE LA UNIVERSIDAD INTERCULTURAL DE TABASCO.

habilidades y actitudes propias de su disciplina, sino aquellas más generales que le permiten la convivencia en la diversidad cultural, sobre todo en tiempos de crisis.

La inequidad reflejada en pobreza, marginación y carencia de medios para el estudio se convierten en serias limitantes durante el proceso de aprendizaje de los estudiantes de zonas rurales y por ende en los resultados de la evaluación académica.

Se prevé haya riesgo de secuelas psicológicas por el confinamiento prolongado y por el alto estrés y frustración por las limitantes para cumplir con los productos evaluables, por lo que se deberá considerar en la atención tutorial y psicopedagógica.

Además, se han obtenido valiosos aprendizajes de esta experiencia, que será compartido con la comunidad estudiantil, personal docente y directivos, para continuar enfrenando el problema de las actividades académicas en confinamiento, puesto que aún no concluye y amenaza con prolongarse en el siguiente ciclo escolar.

Un aspecto positivo a considerar es, que se están generando en todo el mundo de manera rápida, reportes de experiencias, nuevos conocimientos, estrategias y en general nuevas formas de enfrentar el problema y de trabajar en esta pandemia y que puede ser aprovechado en el análisis y toma de decisiones por el colectivo docente.

Ahora más que nunca se valora la importancia de la presencialidad y la participación en aula y campo, propios del modelo educativo intercultural, por lo que se requiere de fuertes dosis de creatividad y voluntad para que este ambiente se continúe a través de los medios electrónicos.

Finalmente, coincidimos con varios de los autores aquí citados en el sentido de que es urgente modificar las instituciones y las políticas educativas para no regresar a la "normalidad" sino abrir nuevos paradigmas educativos que permitan mejorar sustancialmente el acceso a la educación superior e incluyan la interculturalidad como cualidad deseable en todo el sistema educativo; es decir, cumplir a cabalidad el derecho humano a la educación de calidad e incluyente, ese es el reto. 
IMPLICACIONES SOCIOEDUCATIVAS DEL CONFINAMIENTO SOCIAL POR LA PANDEMIA COVID-19. ESTUDIO DE OPINIÓN DE ESTUDIANTES DE LA UNIVERSIDAD INTERCULTURAL DE TABASCO.

\section{REFERENCIAS BIBLIOGRÁFICAS}

Acosta, A. (mayo, 2020). La educación superior ante el COVID-19. Un nuevo reto y viejos resabios. En Reporte CESOP. 2020. COVID-19, la humanidad a prueba, 132. Cámara de Diputados. México. Consultado en: http://www5.diputados.gob.mx/index.php/camara/Centros-de-Estudio/CESOP/TemaCovid-19/Reporte-CESOP.-Covid-19-La-Humanidad-a-Prueba.-Edicion-Especial.-Num.132-mayo-2020

ANUIES. (2020a). Hacia la construcción colectiva de la nueva normalidad en la educación superior. México.

ANUIES. (2020b). Sitio de Espacio Docente: https://espaciodocente.mx/index.html

Balluerka, L. N., Gómez, B. J., Hidalgo, M. M., Goroztiaga, M. A.; Espada, S. J., Padilla, G. J. y Santed, G. M. (2020). Consecuencias psicológicas del COVID-19 y el confinamiento. Universidad del País Vasco. España.

Buendía, E. A. (2020). Desafío de la educación superior en tiempos de pandemia: la contingencia inesperada. En Reporte CESOP. 2020. COVID-19, la humanidad a prueba. Número 132, edición especial, mayo 2020. Cámara de Diputados. México. Consultado en: http://www5.diputados.gob.mx/index.php/camara/Centros-de-Estudio/CESOP/TemaCovid-19/Reporte-CESOP.-Covid-19-La-Humanidad-a-Prueba.-Edicion-Especial.-Num.132-mayo-2020

Castillo, A. M. (2020). Opciones para la actividad escolar durante la pandemia en COVID-19 el caso de Nicaragua, aportes para enfrentar la pandemia. Serie Ciencia, Técnica y Sociedad, Academia de Ciencias de Nicaragua.

Cendales, G. L. y Torres C. A. La Sistematización como experiencia investigativa y formativa. Recuperado de http://www.cepalforja.org/sistem/documentos/lola_cendalesalfonso_torres-la_sistematizacion_como_experiencia_investigativa_y_formativa.pdf.

CEPAL. (2020). América Latina y el Caribe ante la pandemia de COVID-19. Efectos económicos y sociales. Comisión Económica para América Latina y el Caribe. Informe Especial, 1.

CONEVAL. (2018). Medición de la pobreza 2008-2018. Consejo Nacional de Evaluación de la Política de Desarrollo Social. México. https://www.coneval.org.mx/Medicion/MP/Paginas/Pobreza-2018.aspx 
IMPLICACIONES SOCIOEDUCATIVAS DEL CONFINAMIENTO SOCIAL POR LA PANDEMIA COVID-19. ESTUDIO DE OPINIÓN DE ESTUDIANTES DE LA UNIVERSIDAD INTERCULTURAL DE TABASCO.

CONEVAL. (2020). La política social en el contexto de la pandemia por el virus SARS-CoV2 (COVID-19) en México. Consejo Nacional de Evaluación de la Política de Desarrollo Social. México.

Cotino, H. L. (2020). La enseñanza digital en serio y el derecho a la educación en tiempos de coronavirus. Revista de Educación y Derecho, número 21, octubre 2019-marzo 2020. Universidad de Valencia.

Dietz, G. y Mateos, C. L. S. (2020). La interculturalidad educativa en tiempos de pandemia. Muchas sombras y algunas luces. Universidad Veracruzana; en Educar en la Diversidad; Boletín del Grupo de Trabajo Educación e Interculturalidad. CLACSO.

EDUL@Ab. (2020). Decálogo para una docencia online inesperada. Universidad Oberta de Cataluña. http://edulab.uoc.edu/es/2020/03/16/decalogo-para-docencia-lineainesperada-2/

Failache, E., Katzkowicz, N. y Machado, A. (2020). La educación en tiempos de pandemia y el día después: El caso de Uruguay. Revista Internacional de Educación para la Justicia Social. 2020, 9(3e) número extraordinario "Consecuencias del cierre de escuelas por el COVID-19 en las desigualdades educativas.

FAO. (2020). Seguridad alimentaria bajo la pandemia de COVID-19. Organización de las Naciones Unidas para la Alimentación y la Agricultura y Comunidad de Estados Latinoamericanos y caribeños. Recuperado de http://www.fao.org/3/ca8873es/CA8873ES.pdf

Gazzo, M. F. (2020). La educación en tiempos de pandemia COVID-19, nuevas prácticas docentes ¿nuevos estudiantes? RED Sociales; Revista de Departamento de Ciencias Sociales, 07(02). Universidad Nacional de Lujan.

Hooker, B. A. y Castillo, G. L. (2020). La educación superior en contextos multiculturales, visión de futuro. Revista Ciencia e Interculturalidad. Año 13, 26(1).

Landín, M. M. y Sánchez, T. S. (2019). El método biográfico - narrativo. Una herramienta para la investigación educativa. Revista Educación. XXVIII(54). Universidad Veracruzana, pp. 227-242. 
IMPLICACIONES SOCIOEDUCATIVAS DEL CONFINAMIENTO SOCIAL POR LA PANDEMIA COVID-19. ESTUDIO DE OPINIÓN DE ESTUDIANTES DE LA UNIVERSIDAD INTERCULTURAL DE TABASCO.

Mérida, M. Y. y Acuña, G. L.A. (2020). Covid-19, Pobreza y educación en Chiapas: Análisis a los programas educativos emergentes. Revista Internacional de Educación para la Justicia Social. 2020, 9(3e).

OIT. (2020). Los jóvenes y la COVID-19: efectos en los empleos, la educación, los derechos y el bienestar mental. Organización Internacional de Trabajo. Informe de la Encuesta 2020.

Porlan, R. (2020). El cambio de la enseñanza y el aprendizaje en tiempos de pandemia. Revista de Educación Ambiental y Sostenibilidad 2(1) 1502. Universidad de Cádiz.

Ramírez, L. N., Arvizu, V., Ibáñez, L., Claudio, C. y Ramírez, V. (2020). Apoyo ante COVID-19 en Latinoamérica, estudio exploratorio de las necesidades, psico-socio-educativas durante la contingencia. Congreso Internacional Virtual sobre COVID-19, consecuencias psicológicas, sociales, políticas y económicas.

Reimers, F. M. y Schleycher, A. (2020). Un marco para guiar una respuesta educativa a la pandemia del 2020 de COVID-19. En Organización de Estados Iberoamericanos, consultado el 15 de agosto en: https://www.oei.es/Ciencia/Noticia/la-oei-difunde-uninforme-de-la-universidad-de-harvard

Pinilla, S. V. y Lugo, A. N. (2011). Juventud, narrativa y conflicto. Una aproximación al estado del arte de su relación. Revista Latinoamericana de Ciencias sociales, niñez y juventud, 9(2). Julio-diciembre 2011.

Renna, G. H. (2020). El derecho a la educación en tiempos de crisis: alternativas para la continuidad educativa. Universidad Abierta de Recoleta, Universidad Nacional Experimental Samuel Robinson. Clúster de Educación. Caracas Venezuela.

Sparkes, A. C. y Devís, D. J. (2007). Investigación narrativa y su forma de análisis: una visión desde la educación física y el deporte. Recuperado de http://viref.udea.edu.co/contenido/publicaciones/memorias_expo/cuerpo_ciudad/investig acion_narrativa.pdf

Tzoc, J. (2020). Multidimensionalidad en el pensamiento de los pueblos en torno al coronavirus. Universidad Rafael Landívar de Guatemala. Revista Científica Internacional.

UAM. (11 de mayo 2020). El Proyecto de Enseñanza Remota de la UAM, una medida temporal por el COVID-19. $\quad$ Boletín $\quad$ UAM, 271. https://www.uam.mx/ss/s2/comunicacionsocial/boletinesuam/271-20.html 
IMPLICACIONES SOCIOEDUCATIVAS DEL CONFINAMIENTO SOCIAL POR LA PANDEMIA COVID-19. ESTUDIO DE OPINIÓN DE ESTUDIANTES DE LA UNIVERSIDAD INTERCULTURAL DE TABASCO.

Vallejo, I. R. y Álvarez, K. (2020). La pandemia del coronavirus en la Amazonia Ecuatoriana. Vulnerabilidades y olvido del estado. Cuadernos de Campo. Sao Paulo online, 9(1).

Vijil, J. (2020). La educación en Nicaragua: Emergencia más allá del COVID-19; en COVID-19 el caso de Nicaragua, aportes para enfrentar la pandemia. Serie ciencia, técnica y sociedad, Academia de Ciencias de Nicaragua.

Villamizar, E. J. D. y Barbosa, C. J. W. (2017). Sistematización de experiencias. Indicadores y elementos representativos para la investigación educativa. Revista Espacios, 38(47). Año $2017 . \quad$ Recuperado de http://www.revistaespacios.com/a17v38n47/a17v38n47p26.pdf.

Vivanco, Á. A. (2020). Teleeducación en tiempos de COVID-19: brechas de desigualdad. CienciAmérica, 9(2). 